\title{
Existence and uniqueness of solutions for a higher-order coupled fractional differential equations at resonance
}

Lei $\mathrm{Hu}^{1 *}$ and Shuqin Zhang ${ }^{2}$

\section{"Correspondence:}

huleimath@163.com

'School of Science, Shandong

Jiaotong University, Jinan, 250357,

China

Full list of author information is

available at the end of the article

\section{Springer}

\begin{abstract}
In this article, we present the existence of solutions for a higher-order coupled fractional differential equations with the Caputo fractional derivative. Our main approach is the coincidence degree theory due to Mawhin. The most interesting point is the proof of the uniqueness of the solution for the higher-order coupled fractional differential equations at resonance. We give an example to demonstrate our results.
\end{abstract}

MSC: $34 \mathrm{~A} 08 ; 34 \mathrm{~B} 15 ; 34 \mathrm{~F} 15$

Keywords: fractional differential equation; two-point boundary conditions; resonance; coincidence degree

\section{Introduction}

In this article, we study the higher-order coupled fractional differential equation

$$
\begin{cases}D_{0^{+}}^{\alpha} u(t)=f\left(t, v(t), v^{\prime}(t), v^{\prime \prime}(t), \ldots, v^{(N-1)}(t)\right), & 0<t<1, \\ D_{0^{+}}^{\beta} v(t)=g\left(t, u(t), u^{\prime}(t), u^{\prime \prime}(t), \ldots, u^{(N-1)}(t)\right), & 0<t<1,\end{cases}
$$

with the coupled two-point boundary conditions

$$
\begin{cases}u(0)=u^{\prime}(0)=\cdots=u^{(N-2)}=0, & u^{(N-1)}(0)=u^{(N-1)}(1), \\ v(0)=v^{\prime}(0)=\cdots=v^{(N-2)}=0, & v^{(N-1)}(0)=v^{(N-1)}(1),\end{cases}
$$

where $N-1<\alpha, \beta<N, N \geq 2, D_{0^{+}}^{\alpha}$ and $D_{0^{+}}^{\beta}$ denote the Caputo fractional derivative, and $f, g$ are given continuous functions.

Fractional differential equations have been studied extensively. It is caused both by the intensive development of the theory of fractional calculus itself and by the applications such as physics, chemistry, phenomena arising in engineering, economy and science; see e.g. $[1-4]$.

Recently, more and more authors paid attention to the boundary value problems of fractional differential equations; see [5-19]. In [6], the author has investigated the existence of solutions to the coupled systems of fractional differential equations at nonresonance.

(c) $2015 \mathrm{Hu}$ and Zhang. This article is distributed under the terms of the Creative Commons Attribution 4.0 International License (http://creativecommons.org/licenses/by/4.0/), which permits unrestricted use, distribution, and reproduction in any medium, provided you give appropriate credit to the original author(s) and the source, provide a link to the Creative Commons license, and indicate if changes were made. 
Moreover, there have been many works related to the existence of solutions for boundary value problems at resonance; see [10-13, 15-18, 20, 21]. Some papers have dealt with the solutions of multipoint boundary value problems of a coupled fractional differential equations at resonance; see [12, 17].

In [17], Zhang et al. considered a three-point boundary value problem for a coupled system of nonlinear fractional differential equations at resonance given by

$$
\left\{\begin{array}{l}
D_{0^{+}}^{\alpha} u(t)=f\left(t, v(t), D_{0^{+}}^{\beta-1} v(t)\right), \quad 0<t<1, \\
D_{0^{+}}^{\beta} v(t)=g\left(t, u(t), D_{0^{+}}^{\alpha-1} u(t)\right), \quad 0<t<1, \\
u(0)=v(0)=0, \quad u(1)=\sigma_{1} u\left(\eta_{1}\right), \quad v(1)=\sigma_{2} v\left(\eta_{2}\right),
\end{array}\right.
$$

where $1<\alpha, \beta \leq 2,0<\eta_{1}, \eta_{2}<1, \sigma_{1}, \sigma_{2}>0, \sigma_{1} \eta_{1}^{\alpha-1}=\sigma_{2} \eta_{2}^{\beta-1}=1, D$ is Riemann-Liouville fractional derivative, and $f, g:[0,1] \times \mathbb{R}^{2} \rightarrow \mathbb{R}$ are given functions.

In [12], the authors discussed a two-point boundary value problem for a coupled system of fractional differential equations at resonance:

$$
\left\{\begin{array}{l}
D_{0^{+}}^{\alpha} u(t)=f\left(t, v(t), v^{\prime}(t)\right), \quad 0<t<1, \\
D_{0^{+}}^{\beta} v(t)=g\left(t, u(t), u^{\prime}(t)\right), \quad 0<t<1, \\
u(0)=v(0)=0, \quad u^{\prime}(0)=u^{\prime}(1), \quad v^{\prime}(0)=v^{\prime}(1),
\end{array}\right.
$$

where $D_{0^{+}}^{\alpha}, D_{0^{+}}^{\beta}$ is Caputo fractional derivative, $1<\alpha, \beta \leq 2$, and $f, g:[0,1] \times \mathbb{R}^{2} \rightarrow \mathbb{R}$ are given function.

From the above work, we see three facts. Firstly, although the two-point boundary value problems for coupled system of fractional differential equations have been studied by some authors, to the best of our knowledge, higher-order fractional differential equations with the Caputo fractional derivative are seldom considered. Secondly, the nonlinear terms in the equations of this paper satisfy a sublinear growth condition that is weaker than the previous ones (see $[11,15]$ ), meanwhile, the present work generalizes and improves the available results (see $[5,12])$. Thirdly, the uniqueness of the solution is useful for many applications. As far as we know, there are few contributions to the uniqueness of a solution for fractional differential equations. The objective of this paper is to fill the gap in the relevant literature.

The rest of this paper is organized as follows. In Section 2, we give some necessary notations, definitions and lemmas. In Section 3, we study the existence of solutions of (1.1) and (1.2) by the coincidence degree theory due to Mawhin [22]. Finally, an example is given to illustrate our results in Section 4.

\section{Preliminaries}

We present the necessary definitions and lemmas from fractional calculus theory that will be used to prove our main theorems.

Definition 2.1 ([1]) The Riemann-Liouville fractional integral of order $\alpha>0$ of a function $f:(0, \infty) \rightarrow \mathbb{R}$ is given by

$$
I_{0+}^{\alpha} f(t)=\frac{1}{\Gamma(\alpha)} \int_{0}^{t}(t-s)^{\alpha-1} f(s) d s
$$

provided that the right-hand side is pointwise defined on $(0, \infty)$. 
Definition 2.2 ([1]) The Riemann-Liouville fractional derivative of order $\alpha>0$ of a continuous function $f:(0, \infty) \rightarrow \mathbb{R}$ is given by

$$
D_{0^{+}}^{\alpha} f(t)=\frac{1}{\Gamma(n-\alpha)} \int_{0}^{t} \frac{f^{(n)}(s)}{(t-s)^{\alpha-n+1}} d s,
$$

where $n-1<\alpha \leq n$, provided that the right-hand side is pointwise defined on $(0, \infty)$.

Lemma 2.1 ([1]) Let $n-1<\alpha \leq n, u \in C(0,1) \cap L^{1}(0,1)$, then

$$
I_{0+}^{\alpha} D_{0+}^{\alpha} u(t)=u(t)+c_{0}+c_{1} t+\cdots+c_{n-1} t^{n-1},
$$

where $c_{i} \in \mathbb{R}, i=0,1, \ldots, n-1$.

Lemma 2.2 ([1]) If $\beta>0, \alpha+\beta>0$, then the equation

$$
I_{0+}^{\alpha} I_{0+}^{\beta} f(x)=I_{0+}^{\alpha+\beta} f(x),
$$

is satisfied for continuous function $f$.

Now let us recall some notation about the coincidence degree continuation theorem.

Let $Y, Z$ be real Banach spaces, $L: \operatorname{dom} L \subset Y \rightarrow Z$ be a Fredholm map of index zero and $P: Y \rightarrow Y, Q: Z \rightarrow Z$ be continuous projectors such that $\operatorname{ker} L=\operatorname{Im} P, \operatorname{Im} L=\operatorname{ker} Q$, and $Y=\operatorname{ker} L \oplus \operatorname{ker} P, Z=\operatorname{Im} L \oplus \operatorname{Im} Q$. It follows that $\left.L\right|_{\operatorname{dom} L \cap \operatorname{ker} P}: \operatorname{dom} L \cap \operatorname{ker} P \rightarrow \operatorname{Im} L$ is invertible. We denote the inverse of this map by $K_{P}$. If $\Omega$ is an open bounded subset of $Y$, the map $N$ will be called $L$-compact on $\bar{\Omega}$ if $Q N(\bar{\Omega})$ is bounded and $K_{P, Q} N=K_{P}(I-Q) N$ : $\bar{\Omega} \rightarrow Y$ is compact.

Theorem 2.1 Let L be a Fredholm operator of index zero and $N$ be L-compact on $\bar{\Omega}$. Suppose that the following conditions are satisfied:

(1) $L x \neq \lambda N x$ for each $(x, \lambda) \in[(\operatorname{dom} L \backslash \operatorname{ker} L) \cap \partial \Omega] \times(0,1)$;

(2) $N x \notin \operatorname{Im} L$ for each $x \in \operatorname{ker} L \cap \partial \Omega$;

(3) $\operatorname{deg}\left(\left.J Q N\right|_{\operatorname{ker} L}, \Omega \cap \operatorname{ker} L, 0\right) \neq 0$, where $Q: Z \rightarrow Z$ is a continuous projection as above with $\operatorname{Im} L=\operatorname{ker} Q$ and $J: \operatorname{Im} Q \rightarrow \operatorname{ker} L$ is any isomorphism.

Then the equation $L x=N x$ has at least one solution in $\operatorname{dom} L \cap \bar{\Omega}$.

\section{Main results}

In this section, we will prove the existence and uniqueness results for (1.1) and (1.2).

We use the Banach space $E=C[0,1]$ with the norm $\|u\|_{\infty}=\max _{0 \leq t \leq 1}|u(t)|$. We define a linear space $X=\left\{u^{(i)} \in E: i=1,2, \ldots, N-1\right\}$. By means of the linear functional analysis theory, we can prove that $X$ is a Banach space with the norm $\|x\|_{X}=$ $\max \left\{\|u\|_{\infty},\left\|u^{\prime}\right\|_{\infty}, \ldots,\left\|u^{(N-1)}\right\|_{\infty}\right\}$. Further we consider a Banach space $Y=X \times X$ endowed with the norm defined by $\|(u, v)\|_{Y}=\max \left\{\|u\|_{X},\|v\|_{X}\right\}$, and $Z=E \times E$ is a Banach space with the norm defined by $\|(x, y)\|_{Z}=\max \left\{\|x\|_{\infty},\|y\|_{\infty}\right\}$.

Define the linear operator $L_{1}$ from $\operatorname{dom} L_{1} \cap X$ to $E$ by

$$
L_{1} u=D_{0+}^{\alpha} u,
$$

where $\operatorname{dom} L_{1}=\left\{u \in X \mid u^{(i)}(0)=0, u^{(N-1)}(0)=u^{(N-1)}(1), i=0,1, \ldots, N-2\right\}$. 
Define the linear operator $L_{2}$ from $\operatorname{dom} L_{2} \cap X$ to $E$ by

$$
L_{2} v=D_{0+}^{\beta} v
$$

where $\operatorname{dom} L_{2}=\left\{v \in X \mid v^{(i)}(0)=0, v^{(N-1)}(0)=v^{(N-1)}(1), i=0,1, \ldots, N-2\right\}$.

Define the operator $L: \operatorname{dom} L \cap Y \rightarrow Z$ by

$$
L(u, v)=\left(L_{1} u, L_{2} v\right)
$$

where $\operatorname{dom} L=\left\{(u, v) \in Y \mid u \in \operatorname{dom} L_{1}, v \in \operatorname{dom} L_{2}\right\}$, and we define $N: Y \rightarrow Z$ by setting

$$
N(u, v)=\left(N_{1} v, N_{2} u\right),
$$

where $N_{1}: X \rightarrow E$ is defined by

$$
N_{1} v(t)=f\left(t, v(t), v^{\prime}(t), v^{\prime \prime}(t), \ldots, v^{(N-1)}(t)\right)
$$

and $N_{2}: X \rightarrow E$ is defined by

$$
N_{2} u(t)=g\left(t, u(t), u^{\prime}(t), u^{\prime \prime}(t), \ldots, u^{(N-1)}(t)\right)
$$

Then the problem (1.1) and (1.2) can be written by $L(u, v)=N(u, v)$.

Lemma 3.1 The mapping $L: \operatorname{dom} L \subset Y \rightarrow Z$ is a Fredholm operator of index zero.

Proof It is clear that $\operatorname{Ker} L=\left(c_{1} t^{N-1}, c_{2} t^{N-1}\right) \cong \mathbb{R}^{2}$.

Let $(x, y) \in \operatorname{Im} L$, so there exists $(u, v) \in \operatorname{dom} L$ which satisfies $L(u, v)=(x, y)$. By Lemma 2.1, we have

$$
\begin{aligned}
& u(t)=I_{0+}^{\alpha} x(t)+c_{0}+c_{1} t+\cdots+c_{N-1} t^{N-1}, \\
& v(t)=I_{0+}^{\alpha} y(t)+d_{0}+d_{1} t+\cdots+d_{N-1} t^{N-1} .
\end{aligned}
$$

By the definition of $\operatorname{dom} L$, we have $c_{i}=d_{i}=0, i=0,1, \ldots, N-2$. Hence

$$
u(t)=I_{0+}^{\alpha} x(t)+c_{N-1} t^{N-1}, \quad v(t)=I_{0+}^{\beta} y(t)+d_{N-1} t^{N-1} .
$$

According to Lemma 2.2, we get

$$
\begin{aligned}
& u^{(N-1)}(t)=D_{0+}^{N-1}\left(I_{0+}^{\alpha} x(t)+c_{N-1} t^{N-1}\right)=I_{0+}^{\alpha-N+1} x(t)+c_{N-1}(N-1) ! \\
& v^{(N-1)}(t)=D_{0+}^{N-1}\left(I_{0+}^{\beta} y(t)+d_{N-1} t^{N-1}\right)=I_{0+}^{\beta-N+1} y(t)+d_{N-1}(N-1) !
\end{aligned}
$$

Taking into account $u^{(N-1)}(0)=u^{(N-1)}(1)$ and $v^{(N-1)}(0)=v^{(N-1)}(1)$, we obtain

$$
\int_{0}^{1}(1-s)^{\alpha-N} x(s) d s=0, \quad \int_{0}^{1}(1-s)^{\beta-N} y(s) d s=0 .
$$


On the other hand, suppose $(x, y)$ satisfies the above equations. Let $u(t)=I_{0+}^{\alpha} x(t)$ and $v(t)=I_{0+}^{\beta} y(t)$, we can easily prove $(u(t), v(t)) \in \operatorname{dom} L$.

Thus, we conclude that

$$
\operatorname{Im} L=\left\{(x, y) \mid \int_{0}^{1}(1-s)^{\alpha-N} x(s) d s=0 ; \int_{0}^{1}(1-s)^{\beta-N} y(s) d s=0\right\} .
$$

Consider the linear operators $Q_{1}, Q_{2}: E \rightarrow E$ defined by

$$
\begin{aligned}
& Q_{1} x(t)=(\alpha-N+1) \int_{0}^{1}(1-s)^{\alpha-N} x(s) d s, \\
& Q_{2} y(t)=(\beta-N+1) \int_{0}^{1}(1-s)^{\beta-N} y(s) d s .
\end{aligned}
$$

Obviously, $Q(x, y)=\left(Q_{1} x(t), Q_{2} y(t)\right) \cong \mathbb{R}^{2}$. Taking $x(t) \in E$, by a direct computation, we have

$$
\begin{aligned}
Q_{1}\left(Q_{1} x(t)\right) & =Q_{1} x(t) \cdot(\alpha-N+1) \int_{0}^{1}(1-s)^{\alpha-N} d s \\
& =Q_{1} x(t) .
\end{aligned}
$$

Similarly, $Q_{2}^{2}=Q_{2}$. This gives $Q^{2}(x, y)=Q(x, y)$. It is easy to check from $(x, y)=(x, y)-$ $Q(x, y)+Q(x, y)$ that $Z=\operatorname{Im} L+\operatorname{Im} Q$. Moreover, we can see that $Z=\operatorname{Im} L \oplus \operatorname{Im} Q$.

Now, $\operatorname{Ind} L=\operatorname{dim} \operatorname{ker} L-\operatorname{codim} \operatorname{Im} L=0$, and so $L$ is a Fredholm mapping of index zero.

We can define the operators $P_{1}: X \rightarrow X, P_{2}: X \rightarrow X$ and $P:(u, v) \rightarrow\left(P_{1} u, P_{2} v\right)$, where

$$
P_{1} u=\frac{u^{(N-1)}(0)}{(N-1) !} t^{N-1}, \quad P_{2} v=\frac{v^{(N-1)}(0)}{(N-1) !} t^{N-1} .
$$

Obviously, $P_{1}^{2}=P_{1}$ and $P_{2}^{2}=P_{2}$.

Note that

$$
\operatorname{Ker} P=\left\{(u, v) \mid u^{(N-1)}(0)=0, v^{(N-1)}(0)=0\right\} .
$$

Since $(u, v)=(u, v)-P(u, v)+P(u, v)$, it is clear that $Y=\operatorname{Ker} P+\operatorname{Ker} L$. By a simple calculation, we get $\operatorname{Ker} L \cap \operatorname{Ker} P=\{(0,0)\}$. Thus, we get $Y=\operatorname{Ker} L \oplus \operatorname{Ker} P$.

For every $(u, v) \in Y$,

$$
\begin{aligned}
\|P(u, v)\|_{Y} & =\left\|\left(P_{1} u, P_{2} v\right)\right\|_{Y}=\max \left\{\left\|P_{1} u\right\|_{X} ;\left\|P_{2} v\right\|_{X}\right\} \\
& =\max \left\{\frac{\left|u^{(N-1)}(0)\right|}{(N-1) !}\left\|t^{N-1}\right\|_{X} ; \frac{\left|v^{(N-1)}(0)\right|}{(N-1) !}\left\|t^{N-1}\right\|_{X}\right\} \\
& \leq \max \left\{\left|u^{(N-1)}(0)\right| ;\left|v^{(N-1)}(0)\right|\right\} .
\end{aligned}
$$

We define $K_{P}: \operatorname{Im} L \rightarrow \operatorname{dom} L \cap \operatorname{Ker} P$ by $K_{P}(x, y)=\left(I_{0+}^{\alpha} x, I_{0+}^{\beta} y\right)$. 
For $(x, y) \in \operatorname{Im} L$, we have

$$
L K_{P}(x, y)=L\left(I_{0+}^{\alpha} x, I_{0+}^{\beta} y\right)=\left(D_{0+}^{\alpha} I_{0+}^{\alpha} x, D_{0+}^{\beta} I_{0+}^{\beta} y\right)=(x, y)
$$

For $(u, v) \in \operatorname{dom} L \cap \operatorname{Ker} P$, we have $u^{(N-1)}(0)=v^{(N-1)}(0)=0$, so the coefficients $c_{i}$ and $d_{i}$, $i=0,1, \ldots, N-1$, in the expressions

$$
\begin{aligned}
& u=I_{0+}^{\alpha} D_{0+}^{\alpha} u(t)+c_{0}+c_{1} t+\cdots+c_{N-1} t^{N-1}, \\
& v=I_{0+}^{\beta} D_{0+}^{\beta} u(t)+d_{0}+d_{1} t+\cdots+d_{N-1} t^{N-1},
\end{aligned}
$$

are all equal to zero. Thus, we obtain

$$
K_{p} L(x, y)=\left(I_{0+}^{\alpha} D_{0+}^{\alpha} x, I_{0+}^{\beta} D_{0+}^{\beta} y\right)=(x, y) .
$$

That shows that $K_{P}=\left(L_{\operatorname{dom} L \cap \operatorname{Ker} P}\right)^{-1}$.

Again, for each $(x, y) \in \operatorname{Im} L$,

$$
\begin{aligned}
\left\|K_{P}(x, y)\right\|_{Y} & =\left\|\left(I_{0+}^{\alpha} x, I_{0_{+}}^{\beta} y\right)\right\|_{Y}=\max \left\{\left\|I_{0_{+}}^{\alpha} x\right\|_{X^{\prime}} ;\left\|I_{0_{+}}^{\beta} y\right\|_{X}\right\} \\
& \leq \max \left\{\frac{1}{\Gamma(\alpha-N+2)}\|x\|_{\infty} ; \frac{1}{\Gamma(\beta-N+2)}\|y\|_{\infty}\right\} \\
& =\max \left\{a\|x\|_{\infty} ; b\|y\|_{\infty}\right\},
\end{aligned}
$$

where $a=\frac{1}{\Gamma(\alpha-N+2)}, b=\frac{1}{\Gamma(\beta-N+2)}$.

With similar arguments to [5], we obtain the following lemma.

Lemma 3.2 $K_{P}(I-Q) N: Y \rightarrow Y$ is completely continuous.

To obtain our main results, we need the following conditions.

$\left(\mathrm{H}_{1}\right)$ There exist positive constants $a_{1}, a_{2}, b_{i}, c_{i}$, and $\theta_{i}, \lambda_{i} \in[0,1], i=1,2, \ldots, N$, such that for all $\left(x_{1}, x_{2}, \ldots, x_{N}\right) \in \mathbb{R}^{N}$,

$$
\begin{array}{ll}
\left|f\left(t, x_{1}, x_{2}, \ldots, x_{N}\right)\right| \leq a_{1}+b_{1}\left|x_{1}\right|^{\theta_{1}}+b_{2}\left|x_{2}\right|^{\theta_{2}}+\cdots+b_{N}\left|x_{N}\right|^{\theta_{N}}, \quad \forall t \in[0,1], \\
\left|g\left(t, x_{1}, x_{2}, \ldots, x_{N}\right)\right| \leq a_{2}+c_{1}\left|x_{1}\right|^{\lambda_{1}}+c_{2}\left|x_{2}\right|^{\lambda_{2}}+\cdots+c_{N}\left|x_{N}\right|^{\lambda_{N}}, \quad \forall t \in[0,1] .
\end{array}
$$

$\left(\mathrm{H}_{2}\right)$ There exists a constant $A>0$ such that for any $c_{1}, c_{2} \in \mathbb{R}^{2}$, if $\min \left\{\left|c_{1}\right|,\left|c_{2}\right|\right\}>A$, one has either

$$
c_{1} \cdot N_{1}\left(c_{2} t^{N-1}\right)>0, \quad c_{2} \cdot N_{2}\left(c_{1} t^{N-1}\right)>0
$$

or

$$
c_{1} \cdot N_{1}\left(c_{2} t^{N-1}\right)<0, \quad c_{2} \cdot N_{2}\left(c_{1} t^{N-1}\right)<0 .
$$

$\left(\mathrm{H}_{3}\right) \max \left\{2 a \sum_{i=1}^{N} b_{i}, a \sum_{i=1}^{N} b_{i}+b \sum_{i=1}^{N} c_{i}, 2 b \sum_{i=1}^{N} c_{i}\right\}<1$. 
Lemma 3.3 $\Omega_{1}=\{(u, v) \in \operatorname{dom} L \backslash \operatorname{Ker} L: L(u, v)=\lambda N(u, v), \lambda \in[0,1]\}$ is bounded.

Proof For $(u, v) \in \Omega_{1}$, thus $\lambda \neq 0$. Also, $L(u, v)=\lambda N(u, v) \in \operatorname{Im} L=\operatorname{Ker} Q$, that is,

$$
\begin{aligned}
& \lambda(\alpha-N+1) \int_{0}^{1}(1-s)^{\alpha-N} f\left(s, v(s), v^{\prime}(s), v^{\prime \prime}(s), \ldots, v^{(N-1)}(s)\right) d s=0, \\
& \lambda(\beta-N+1) \int_{0}^{1}(1-s)^{\beta-N} g\left(s, u(s), u^{\prime}(s), u^{\prime \prime}(s), \ldots, u^{(N-1)}(s)\right) d s=0 .
\end{aligned}
$$

By the integral mean value theorem, there exist $t_{0}, t_{1} \in[0,1]$ such that

$$
\begin{aligned}
& f\left(t_{0}, v\left(t_{0}\right), v^{\prime}\left(t_{0}\right), v^{\prime \prime}\left(t_{0}\right), \ldots, v^{(N-1)}\left(t_{0}\right)\right)=0, \\
& g\left(t_{1}, u\left(t_{1}\right), u^{\prime}\left(t_{1}\right), u^{\prime \prime}\left(t_{1}\right), \ldots, u^{(N-1)}\left(t_{1}\right)\right)=0 .
\end{aligned}
$$

From $\left(\mathrm{H}_{2}\right)$, we get $\left|u^{(N-1)}\left(t_{1}\right)\right| \leq A$ and $\left|v^{(N-1)}\left(t_{0}\right)\right| \leq A$.

Again for $(u, v) \in \Omega_{1},(u, v) \in \operatorname{dom}(L) \backslash \operatorname{Ker}(L)$, then $(I-P)(u, v) \in \operatorname{dom} L \cap \operatorname{Ker} P$ and $L P(u, v)=(0,0)$, thus from (3.2), we have

$$
\begin{aligned}
\|(I-P)(u, v)\|_{Y} & =\left\|K_{P} L(I-P)(u, v)\right\|_{Y}=\left\|K_{P}\left(L_{1} u, L_{2} v\right)\right\|_{Y} \\
& \leq \max \left\{a\left\|N_{1} v\right\|_{\infty} ; b\left\|N_{2} u\right\|_{\infty}\right\} .
\end{aligned}
$$

By $L u=\lambda N u$ and $u \in \operatorname{dom} L$, we have

$$
\begin{aligned}
u(t)= & \frac{1}{\Gamma(\alpha)} \int_{0}^{t}(t-s)^{\alpha-1} f\left(s, v(s), \ldots, v^{(N-1)}(s)\right) d s \\
& -u(0)-u^{\prime}(0) t-\cdots-\frac{u^{(N-1)}(0)}{(N-1) !} t^{N-1} .
\end{aligned}
$$

Furthermore, we have

$$
u^{(N-1)}(t)=\frac{1}{\Gamma(\alpha-N+1)} \int_{0}^{t}(t-s)^{\alpha-N} f\left(s, v(s), \ldots, v^{(N-1)}(s)\right) d s-u^{(N-1)}(0) .
$$

Substituting $t=t_{1}$ into the above equation, we get

$$
u^{(N-1)}\left(t_{1}\right)=\frac{1}{\Gamma(\alpha-N+1)} \int_{0}^{t_{1}}\left(t_{1}-s\right)^{\alpha-N} f\left(s, v(s), \ldots, v^{(N-1)}(s)\right) d s-u^{(N-1)}(0) .
$$

Together with $\left|u^{(N-1)}\left(t_{1}\right)\right| \leq A$, we derive that

$$
\begin{aligned}
\left|u^{(N-1)}(0)\right| \leq & \left|\frac{1}{\Gamma(\alpha-N+1)} \int_{0}^{t_{1}}\left(t_{1}-s\right)^{\alpha-N} f\left(s, v(s), \ldots, v^{(N-1)}(s)\right) d s\right| \\
& +\left|u^{(N-1)}\left(t_{1}\right)\right| \\
\leq & A+\frac{1}{\Gamma(\alpha-N+1)} \int_{0}^{t_{1}}\left(t_{1}-s\right)^{\alpha-N}\left|f\left(s, v(s), \ldots, v^{(N-1)}(s)\right)\right| d s \\
\leq & A+\frac{1}{\Gamma(\alpha-N+1)} \int_{0}^{t_{1}}\left(t_{1}-s\right)^{\alpha-N}\left(a_{1}+\sum_{i=1}^{N} b_{i}\left|v^{(i-1)}\right|^{\theta_{i}}\right) d s
\end{aligned}
$$




$$
\begin{aligned}
& \leq A+\frac{1}{\Gamma(\alpha-N+1)}\left(a_{1}+\sum_{i=1}^{N} b_{i}\left\|v^{(i-1)}\right\|_{\infty}^{\theta_{i}}\right) \cdot \int_{0}^{t_{1}}\left(t_{1}-s\right)^{\alpha-N} d s \\
& \leq A+a a_{1}+a \sum_{i=1}^{N} b_{i}\left\|v^{(i-1)}\right\|_{\infty}^{\theta_{i}} .
\end{aligned}
$$

With similar arguments, we obtain

$$
\left|v^{(N-1)}(0)\right| \leq A+a_{2} b+b \sum_{i=1}^{N} c_{i}\left\|u^{(i-1)}\right\|_{\infty}^{\lambda_{i}}
$$

From (3.1) and (3.3), we have

$$
\begin{aligned}
\|(u, v)\|_{Y}= & \|P(u, v)+(I-P)(u, v)\|_{Y} \\
\leq & \|P(u, v)\|_{Y}+\|(I-P)(u, v)\|_{Y} \\
\leq & \max \left\{\left|u^{(N-1)}(0)\right|+a\left\|N_{1} v\right\|_{\infty},\left|u^{(N-1)}(0)\right|+b\left\|N_{2} u\right\|_{\infty},\right. \\
& \left.\left|v^{(N-1)}(0)\right|+a\left\|N_{1} v\right\|_{\infty},\left|v^{(N-1)}(0)\right|+b\left\|N_{2} u\right\|_{\infty}\right\} .
\end{aligned}
$$

In what follows, the proof can be divided into four cases.

Case 1. $\|(u, v)\|_{Y} \leq\left|u^{(N-1)}(0)\right|+a\left\|N_{1} v\right\|_{\infty}$.

By (3.4) and $\left(\mathrm{H}_{1}\right)$, we have

$$
\begin{aligned}
\|(u, v)\|_{Y} & \leq\left|u^{(N-1)}(0)\right|+a\left\|N_{1} v\right\|_{\infty} \\
& \leq A+a a_{1}+a \sum_{i=1}^{N} b_{i}\left\|v^{(i-1)}\right\|_{\infty}^{\theta_{i}}+a\left\|N_{1} v\right\|_{\infty} \\
& \leq A+a a_{1}+a \sum_{i=1}^{N} b_{i}\left\|v^{(i-1)}\right\|_{\infty}^{\theta_{i}}+a\left\|f\left(t, v(t), v^{\prime}(t), \ldots, v^{(N-1)}(t)\right)\right\|_{\infty} \\
& \leq A+2 a a_{1}+a \sum_{i=1}^{N} b_{i}\left\|v^{(i-1)}\right\|_{\infty}^{\theta_{i}}+a \sum_{i=1}^{N} b_{i}\left\|v^{(i-1)}\right\|_{\infty}^{\theta_{i}} \\
& =A+2 a a_{1}+2 a \sum_{i=1}^{N} b_{i}\left\|v^{(i-1)}\right\|_{\infty}^{\theta_{i}} .
\end{aligned}
$$

According to $\left(\mathrm{H}_{3}\right)$ and the definition of $\|(u, v)\|_{Y}$, we can derive $\|v\|_{X}$ are bounded. Therefore $\Omega_{1}$ is bounded.

Case 2. $\|(u, v)\|_{Y} \leq\left|v^{(N-1)}(0)\right|+b\left\|N_{2} u\right\|_{\infty}$. The proof is similar to Case 1. Here, we omit it.

Case 3. $\|(u, v)\|_{Y} \leq\left|u^{(N-1)}(0)\right|+b\left\|N_{2} u\right\|_{\infty}$.

From (3.4) and $\left(\mathrm{H}_{1}\right)$, we obtain

$$
\begin{aligned}
\|(u, v)\|_{Y} & \leq\left|u^{(N-1)}(0)\right|+b\left\|N_{2} u\right\|_{\infty} \\
& \leq A+a a_{1}+a \sum_{i=1}^{N} b_{i}\left\|v^{(i-1)}\right\|_{\infty}^{\theta_{i}}+b\left\|N_{2} u\right\|_{\infty}
\end{aligned}
$$




$$
\begin{aligned}
& \leq A+a a_{1}+a \sum_{i=1}^{N} b_{i}\left\|v^{(i-1)}\right\|_{\infty}^{\theta_{i}}+b\left\|g\left(t, u(t), u^{\prime}(t), \ldots, u^{(N-1)}(t)\right)\right\|_{\infty} \\
& \leq A+a a_{1}+b a_{2}+a \sum_{i=1}^{N} b_{i}\left\|v^{(i-1)}\right\|_{\infty}^{\theta_{i}}+b \sum_{i=1}^{N} c_{i}\left\|u^{(i-1)}\right\|_{\infty}^{\lambda_{i}} .
\end{aligned}
$$

By $\left(\mathrm{H}_{3}\right)$, we easily conclude that $\|(u, v)\|_{Y}$ is bounded. Therefore $\Omega_{1}$ is bounded.

Case 4. $\|(u, v)\|_{\infty} \leq\left|v^{(N-1)}(0)\right|+a\left\|N_{1} v\right\|_{\infty}$. The proof is similar to the Case 2 . Here, we omit it.

According to the above arguments, we prove that $\Omega_{1}$ is bounded.

Lemma 3.4 $\Omega_{2}=\{(u, v) \in \operatorname{Ker} L: N(u, v) \in \operatorname{Im} L\}$ is bounded.

Proof Let $(u, v) \in \operatorname{Ker} L$, so we have $u=c_{1} t^{N-1}, v=c_{2} t^{N-1}, c_{1}, c_{2} \in \mathbb{R}$. In view of $N(u, v)=$ $\left(N_{1} v, N_{2} u\right) \in \operatorname{Im} L=\operatorname{Ker} Q$, we have

$$
\begin{aligned}
& \int_{0}^{1}(1-s)^{\alpha-N} f\left(t, c_{2} t^{N-1},(N-1) c_{2} t^{N-2}, \ldots, c_{2}(N-1) !\right) d t=0 \\
& \int_{0}^{1}(1-s)^{\beta-N} g\left(t, c_{1} t^{N-1},(N-1) c_{1} t^{N-2}, \ldots, c_{1}(N-1) !\right) d t=0 .
\end{aligned}
$$

By the integral mean value theorem, there exist constants $t_{0}, t_{1} \in[0,1]$ such that

$$
\begin{aligned}
& f\left(t_{0}, c_{2} t_{0}^{N-1},(N-1) c_{2} t_{0}^{N-2}, \ldots, c_{2}(N-1) !\right)=0, \\
& g\left(t_{1}, c_{1} t_{1}^{N-1},(N-1) c_{1} t_{1}^{N-2}, \ldots, c_{1}(N-1) !\right)=0,
\end{aligned}
$$

which together with $\left(\mathrm{H}_{2}\right)$ imply $\left|c_{i}\right| \leq \frac{A}{(N-1) !}, i=1,2$. Hence, $\Omega_{2}$ is bounded.

Lemma 3.5 $\Omega_{3}=\{(u, v) \in \operatorname{Ker} L: \lambda(u, v)+(1-\lambda) Q N(u, v)=(0,0), \lambda \in[0,1]\}$ is bounded.

Proof Let $(u, v) \in \operatorname{Ker} L$, so we have $u=c_{1} t^{N-1}, v=c_{2} t^{N-1}, c_{1}, c_{2} \in \mathbb{R}$, and

$$
\lambda c_{1} t^{N-1}+(1-\lambda) Q_{1} N_{1}(v)=0, \quad \lambda c_{2} t^{N-1}+(1-\lambda) Q_{2} N_{2}(u)=0,
$$

that is to say,

$$
\begin{aligned}
& \lambda c_{1} t^{N-1}+(1-\lambda) \int_{0}^{1} f\left(t, c_{2} t^{N-1}, c_{2}(N-1) t^{N-2}, \ldots, c_{2}(N-1) !\right) d t=0, \\
& \lambda c_{2} t^{N-1}+(1-\lambda) \int_{0}^{1} g\left(t, c_{1} t^{N-1}, c_{1}(N-1) t^{N-2}, \ldots, c_{1}(N-1) !\right) d t=0 .
\end{aligned}
$$

If $\lambda=0$, then $\left|c_{i}\right| \leq \frac{A}{(N-1) !}, i=1,2$. If $\lambda \in(0,1]$, then we can have $\left|c_{i}\right| \leq \frac{A}{(N-1) !}, i=1,2$. Otherwise, if $\left|c_{i}\right|>\frac{A}{(N-1) !}, i=1,2$, in view of the first part of $\left(\mathrm{H}_{2}\right)$, one has

$$
\begin{aligned}
& \lambda c_{1}^{2} t^{N-1}+(1-\lambda) \int_{0}^{1} c_{1} f\left(t, c_{2} t^{N-1}, c_{2}(N-1) t^{N-2}, \ldots, c_{2}(N-1) !\right) d t>0, \\
& \lambda c_{2}^{2} t^{N-1}+(1-\lambda) \int_{0}^{1} c_{2} g\left(t, c_{1} t^{N-1}, c_{1}(N-1) t^{N-2}, \ldots, c_{1}(N-1) !\right) d t>0,
\end{aligned}
$$

which contradict (3.6) and (3.7). Thus, $\Omega_{3}$ is bounded. 
Remark 3.1 If the second part of $\left(\mathrm{H}_{2}\right)$ holds, then the set

$$
\Omega_{3}^{\prime}=\{(u, v) \in \operatorname{Ker} L:-\lambda(u, v)+(1-\lambda) Q N(u, v)=(0,0), \lambda \in[0,1]\}
$$

is bounded.

Theorem 3.1 Suppose $\left(\mathrm{H}_{1}\right)-\left(\mathrm{H}_{3}\right)$ hold, then the problem (1.1) and (1.2) has at least on solution in $Y$.

Proof Let $\Omega$ to be a bounded open subset of $Y$, such that $\bigcup_{i=1}^{3} \bar{\Omega}_{i} \subset \Omega$. It follows from Lemma 3.2 that $N$ is $L$-compact on $\Omega$. By Lemma 3.3, Lemma 3.4, and Lemma 3.5, we get:

(1) $L u \neq \lambda N u$, for every $(u, v, \lambda) \in[(\operatorname{dom} L \backslash \operatorname{Ker} L) \cap \partial \Omega] \times(0,1)$.

(2) $N u \notin \operatorname{Im} L$ for every $u \in \operatorname{Ker} L \cap \partial \Omega$.

(3) Let $H((u, v), \lambda)= \pm \lambda I(u, v)+(1-\lambda) J Q N(u, v)$, where $I$ is the identical operator. Via the homotopy property of the degree, we obtain

$$
\begin{aligned}
\operatorname{deg}\left(\left.J Q N\right|_{\operatorname{ker} L}, \Omega \cap \operatorname{ker} L, 0\right) & =\operatorname{deg}(H(\cdot, 0), \Omega \cap \operatorname{ker} L, 0) \\
& =\operatorname{deg}(H(\cdot, 1), \Omega \cap \operatorname{ker} L, 0) \\
& =\operatorname{deg}(I, \Omega \cap \operatorname{ker} L, 0)=1 \neq 0 .
\end{aligned}
$$

Applying Theorem 2.1, we conclude that $L(u, v)=N(u, v)$ has at least one solution in $\operatorname{dom} L \cap \bar{\Omega}$.

Under the stronger conditions imposed on $f$, we can prove the uniqueness of the solutions to BVP (1.1) and (1.2).

Theorem 3.2 Suppose the condition $\left(\mathrm{H}_{1}\right)$ in Theorem 2.1 is replaced by the following conditions:

$\left(\mathrm{H}_{1}^{\prime}\right)$ There exist positive constants $a_{i}, b_{i}, i=0,1, \ldots, N-1$, such that for all $\left(x_{1}, x_{2}, \ldots, x_{N}\right)$, $\left(y_{1}, y_{2}, \ldots, y_{N}\right) \in \mathbb{R}^{N}$ one has

$$
\begin{aligned}
& \left|f\left(t, x_{1}, x_{2}, \ldots, x_{N}\right)-f\left(t, y_{1}, y_{2}, \ldots, y_{N}\right)\right| \leq a_{0}\left|x_{1}-y_{1}\right|+\cdots+a_{N-1}\left|x_{N}-y_{N}\right| \\
& \left|g\left(t, x_{1}, x_{2}, \ldots, x_{N}\right)-g\left(t, y_{1}, y_{2}, \ldots, y_{N}\right)\right| \leq b_{0}\left|x_{1}-y_{1}\right|+\cdots+b_{N-1}\left|x_{N}-y_{N}\right| .
\end{aligned}
$$

$\left(\mathrm{H}_{1}^{\prime \prime}\right)$ There exist positive constants $k_{i}, l_{i}, i=0,1, \ldots, N-1$, such that for all $\left(x_{1}, x_{2}, \ldots, x_{N}\right)$, $\left(y_{1}, y_{2}, \ldots, y_{N}\right) \in \mathbb{R}^{N}$, one has

$$
\begin{aligned}
& \left|f\left(t, x_{1}, x_{2}, \ldots, x_{N}\right)-f\left(t, y_{1}, y_{2}, \ldots, y_{N}\right)\right| \\
& \quad \geq l_{N-1}\left|x_{N}-y_{N}\right|-l_{0}\left|x_{1}-y_{1}\right|-l_{1}\left|x_{2}-y_{2}\right|-\cdots-l_{N-2}\left|x_{N-1}-y_{N-1}\right|, \\
& \left|g\left(t, x_{1}, x_{2}, \ldots, x_{N}\right)-g\left(t, y_{1}, y_{2}, \ldots, y_{N}\right)\right| \\
& \quad \geq k_{N-1}\left|x_{N}-y_{N}\right|-k_{0}\left|x_{1}-y_{1}\right|-k_{1}\left|x_{2}-y_{2}\right|-\cdots-k_{N-2}\left|x_{N-1}-y_{N-1}\right| .
\end{aligned}
$$


Then BVP (1.1) and (1.2) has a unique solution, provided that

$$
\begin{aligned}
& \max \left\{p_{1}+q_{1}+2 r_{2}, p_{2}+q_{2}+2 r_{1},\right. \\
& \left.p_{1}+q_{1}+r_{1}+r_{2}, p_{2}+q_{2}+r_{1}+r_{2}\right\}<1,
\end{aligned}
$$

where $p_{1}=\frac{l_{0}}{l_{N-1}}, p_{2}=\frac{k_{0}}{k_{N-1}}, q_{1}=\sum_{i=1}^{N-2} \frac{l_{i}}{l_{N-1}}, q_{2}=\sum_{i=1}^{N-2} \frac{k_{i}}{k_{N-1}}, r_{1}=a \sum_{i=0}^{N-1} a_{i}, r_{2}=b \sum_{i=0}^{N-1} b_{i}$.

Proof We let $y_{i}=0, i=1,2, \ldots, N, a_{1}=\max _{t \in[0,1]}|f(t, 0, \ldots, 0)|$ and $a_{2}=\max _{t \in[0,1]} \mid g(t, 0$, $\ldots, 0) \mid$, then from (3.8) we can show that the condition $\left(\mathrm{H}_{1}\right)$ is satisfied. Therefore, the existence of a solution for the coupled system (1.1) and (1.2) follows from Theorem 3.1.

Suppose $\left(u_{i}, v_{i}\right) \in Y, i=1,2$ are two solutions of BVP (1.1) and (1.2), then

$$
\begin{aligned}
& D_{0^{+}}^{\alpha} u_{i}(t)=f\left(t, v_{i}(t), v_{i}^{\prime}(t), \ldots, v_{i}^{(N-1)}(t)\right), \\
& D_{0^{+}}^{\beta} v_{i}(t)=g\left(t, u_{i}(t), u_{i}^{\prime}(t), \ldots, u_{i}^{(N-1)}(t)\right) .
\end{aligned}
$$

Note $u=u_{1}-u_{2}, v=v_{1}-v_{2}$, thus we have the following equations:

$$
\begin{aligned}
& D_{0^{+}}^{\alpha} u(t)=f\left(t, v_{1}(t), v_{1}^{\prime}(t), \ldots, v_{1}^{(N-1)}(t)\right)-f\left(t, v_{2}(t), v_{2}^{\prime}(t), \ldots, v_{2}^{(N-1)}(t)\right), \\
& D_{0^{+}}^{\beta} v(t)=g\left(t, u_{1}(t), u_{1}^{\prime}(t), \ldots, u_{1}^{(N-1)}(t)\right)-g\left(t, u_{2}(t), u_{2}^{\prime}(t), \ldots, u_{2}^{(N-1)}(t)\right) .
\end{aligned}
$$

By $\operatorname{Im} L=\operatorname{Ker} Q$, we have

$$
\begin{aligned}
& \int_{0}^{1}(1-s)^{\alpha-N} f\left(t, v_{1}(t), v_{1}^{\prime}(t), \ldots, v_{1}^{(N-1)}(t)\right)-f\left(t, v_{2}(t), v_{2}^{\prime}(t), \ldots, v_{2}^{(N-1)}(t)\right) d t=0, \\
& \int_{0}^{1}(1-s)^{\beta-N} g\left(t, u_{1}(t), u_{1}^{\prime}(t), \ldots, u_{1}^{(N-1)}(t)\right)-g\left(t, u_{2}(t), u_{2}^{\prime}(t), \ldots, u_{2}^{(N-1)}(t)\right) d t=0 .
\end{aligned}
$$

By the integral mean value theorem, there exist $\xi, \eta \in[0,1]$, such that

$$
\begin{aligned}
& f\left(\xi, v_{1}(\xi), v_{1}^{\prime}(\xi), \ldots, v_{1}^{(N-1)}(\xi)\right)-f\left(\xi, v_{2}(\xi), v_{2}^{\prime}(\xi), \ldots, v_{2}^{(N-1)}(\xi)\right)=0, \\
& g\left(\eta, u_{1}(\eta), u_{1}^{\prime}(\eta), \ldots, u_{1}^{(N-1)}(\eta)\right)-g\left(\eta, u_{2}(\eta), u_{2}^{\prime}(\eta), \ldots, u_{2}^{(N-1)}(\eta)\right)=0 .
\end{aligned}
$$

By $\left(\mathrm{H}_{1}^{\prime \prime}\right)$, we have

$$
\begin{aligned}
0 & =\left|f\left(\xi, v_{1}(\xi), v_{1}^{\prime}(\xi), \ldots, v_{1}^{(N-1)}(\xi)\right)-f\left(\xi, v_{2}(\xi), v_{2}^{\prime}(\xi), \ldots, v_{2}^{(N-1)}(\xi)\right)\right| \\
& \geq l_{N-1}\left|v^{(N-1)}(\xi)\right|-l_{0}|v(\xi)|-l_{1}\left|v^{\prime}(\xi)\right|-\cdots-l_{N-2}\left|v^{(N-2)}(\xi)\right| \\
0 & =\left|g\left(\eta, u_{1}(\eta), u_{1}^{\prime}(\eta), \ldots, u_{1}^{(N-1)}(\eta)\right)-g\left(\eta, u_{2}(\eta), u_{2}^{\prime}(\eta), \ldots, u_{2}^{(N-1)}(\eta)\right)\right| \\
& \geq k_{N-1}\left|u^{(N-1)}(\eta)\right|-k_{0}|u(\eta)|-k_{1}\left|u^{\prime}(\eta)\right|-\cdots-k_{N-2}\left|u^{(N-2)}(\eta)\right| .
\end{aligned}
$$

It follows from the two inequalities above that

$$
\begin{aligned}
\left|v^{(N-1)}(\xi)\right| & \leq \frac{l_{0}}{l_{N-1}}|v(\xi)|+\frac{l_{1}}{l_{N-1}}\left|v^{\prime}(\xi)\right|+\cdots+\frac{l_{N-2}}{l_{N-1}}\left|v^{(N-2)}(\xi)\right| \\
& \leq \frac{l_{0}}{l_{N-1}}\|v\|_{\infty}+\sum_{i=1}^{N-2} \frac{l_{i}}{l_{N-1}}\left\|v^{(i)}\right\|_{\infty} \\
& \leq\left(p_{1}+q_{1}\right)\|v\|_{X}
\end{aligned}
$$


and

$$
\begin{aligned}
\left|u^{(N-1)}(\eta)\right| & \leq \frac{k_{0}}{k_{N-1}}\|u\|_{\infty}+\sum_{i=1}^{N-2} \frac{k_{i}}{k_{N-1}}\left\|u^{(i)}\right\|_{\infty} \\
& \leq\left(p_{2}+q_{2}\right)\|u\|_{X} .
\end{aligned}
$$

By (3.9), we obtain

$$
\begin{aligned}
u^{(N-1)}(t)= & I_{0+}^{\alpha-(N-1)}\left[f\left(t, v_{1}(t), v_{1}^{\prime}(t), \ldots, v_{1}^{(N-1)}(t)\right)\right. \\
& \left.-f\left(t, v_{2}(t), v_{2}^{\prime}(t), \ldots, v_{2}^{(N-1)}(t)\right)\right]-u^{(N-1)}(0) .
\end{aligned}
$$

Substituting $t=\eta$ into the above equation, we get

$$
\begin{aligned}
u^{(N-1)}(\eta)= & I_{0+}^{\alpha-(N-1)}\left[f\left(t, v_{1}(t), v_{1}^{\prime}(t), \ldots, v_{1}^{(N-1)}(t)\right)\right. \\
& \left.-f\left(t, v_{2}(t), v_{2}^{\prime}(t), \ldots, v_{2}^{(N-1)}(t)\right)\right]_{t=\eta}-u^{(N-1)}(0) .
\end{aligned}
$$

By $\left(\mathrm{H}_{1}^{\prime}\right),(3.10)$ and the definition of $\|v\|_{X}$, we have

$$
\begin{aligned}
\left|u^{(N-1)}(0)\right| & \leq\left|u^{(N-1)}(\eta)\right|+\frac{1}{\Gamma(\alpha-N+1)} \int_{0}^{\eta}(\eta-s)^{\alpha-N} d s \cdot \sum_{i=0}^{N-1} a_{i}\left\|v^{(i)}\right\|_{\infty} \\
& \leq\left|u^{(N-1)}(\eta)\right|+\|v\|_{X} \cdot \frac{1}{\Gamma(\alpha-N+1)} \int_{0}^{\eta}(\eta-s)^{\alpha-N} d s \cdot \sum_{i=0}^{N-1} a_{i} \\
& \leq\left(p_{2}+q_{2}\right)\|u\|_{X}+\|v\|_{X} \cdot a \sum_{i=0}^{N-1} a_{i} \\
& =\left(p_{2}+q_{2}\right)\|u\|_{X}+r_{1}\|v\|_{X} .
\end{aligned}
$$

Similarly,

$$
\begin{aligned}
\left|v^{(N-1)}(0)\right| & \leq \frac{l_{0}}{l_{1}}\|v\|_{\infty}+\sum_{i=2}^{N-1} \frac{l_{i}}{l_{1}}\left\|v^{(i-1)}\right\|_{\infty}+\|u\|_{X} \cdot b \sum_{i=0}^{N-1} b_{i} \\
& =\left(p_{1}+q_{1}\right)\|v\|_{X}+r_{2}\|u\|_{X} .
\end{aligned}
$$

According to (3.3), (3.11), and (3.12), we have

$$
\begin{aligned}
\|(u, v)\|_{Y}= & \|P(u, v)+(I-P)(u, v)\|_{Y} \leq\|P(u, v)\|_{Y}+\|(I-P)(u, v)\|_{Y} \\
= & \max \left\{\left|u^{(N-1)}(0)\right| ;\left|v^{(N-1)}(0)\right|\right\}+\max \left\{a\left\|L_{1} u\right\|_{\infty} ; b\left\|L_{2} v\right\|_{\infty}\right\} \\
\leq & \max \left\{\left(p_{2}+q_{2}\right)\|u\|_{X}+r_{1}\|v\|_{X}+a\left\|L_{1} u\right\|_{\infty},\right. \\
& \left(p_{2}+q_{2}\right)\|u\|_{X}+r_{1}\|v\|_{X}+b\left\|L_{2} v\right\|_{\infty} \\
& \left(p_{1}+q_{1}\right)\|v\|_{X}+r_{2}\|u\|_{X}+a\left\|L_{1} u\right\|_{\infty} \\
& \left.\left(p_{1}+q_{1}\right)\|v\|_{X}+r_{2}\|u\|_{X}+b\left\|L_{2} v\right\|_{\infty}\right\} .
\end{aligned}
$$

Our proof can be divided into four cases. 
Case 1. $\|(u, v)\|_{Y} \leq\left(p_{2}+q_{2}\right)\|u\|_{X}+r_{1}\|v\|_{X}+a\left\|L_{1} u\right\|_{\infty}$.

By $\left(\mathrm{H}_{1}^{\prime}\right)$ and the definition of $\|(u, v)\|_{Y}$, we have

$$
\begin{aligned}
\|(u, v)\|_{Y} & \leq\left(p_{2}+q_{2}\right)\|u\|_{X}+r_{1}\|v\|_{X}+a\left\|L_{1} u\right\|_{\infty} \\
& \leq\left(p_{2}+q_{2}\right)\|u\|_{X}+r_{1}\|v\|_{X}+a\left(a_{0}\|v\|_{\infty}+\sum_{i=1}^{N-1} a_{i}\left\|v^{(i)}\right\|_{\infty}\right) \\
& \leq\left(p_{2}+q_{2}\right)\|u\|_{X}+2 r_{1}\|v\|_{X} \\
& \leq\left(p_{2}+q_{2}+2 r_{1}\right)\|(u, v)\|_{Y} .
\end{aligned}
$$

By the assumption (3.8), the coefficient on the right side of (3.13) is less than 1. So we have $\|u\|_{X}=\|v\|_{X}=0$, i.e., $u_{1}=u_{2}, v_{1}=v_{2}$.

Case 2. $\|(u, v)\|_{Y} \leq\left(p_{1}+q_{1}\right)\|v\|_{X}+r_{2}\|u\|_{X}+b\left\|L_{2} v\right\|_{\infty}$. The proof is similar to Case 1 . So we omit it.

Case 3. $\|(u, v)\|_{\infty} \leq\left(p_{2}+q_{2}\right)\|u\|_{X}+r_{1}\|v\|_{X}+b\left\|L_{2} v\right\|_{\infty}$.

By $\left(\mathrm{H}_{1}^{\prime}\right)$ and the definition of $\|(u, v)\|_{Y}$, we have

$$
\begin{aligned}
\|(u, v)\|_{Y} & \leq\left(p_{2}+q_{2}\right)\|u\|_{X}+r_{1}\|v\|_{X}+b\left\|L_{2} v\right\|_{\infty} \\
& \leq\left(p_{2}+q_{2}\right)\|u\|_{X}+r_{1}\|v\|_{X}+b\left(b_{0}\|u\|_{\infty}+\sum_{i=1}^{N-1} b_{i}\left\|u^{(i)}\right\|_{\infty}\right) \\
& \leq\left(p_{2}+q_{2}+r_{2}\right)\|u\|_{X}+r_{1}\|v\|_{X} \\
& \leq\left(p_{2}+q_{2}+r_{1}+r_{2}\right)\|(u, v)\|_{Y} .
\end{aligned}
$$

By our assumption (3.8), the coefficients on the right side of (3.14) are all less than 1. So we have $\|u\|=\|v\|=0$, so that $u_{1}=u_{2}, v_{1}=v_{2}$.

Case 4. $\|(u, v)\|_{Y} \leq\left(p_{1}+q_{1}\right)\|v\|_{X}+r_{2}\|u\|_{X}+a\left\|L_{1} u\right\|_{\infty}$. The proof is similar to Case 3. Here, we omit it.

By the above argument, we have derived that BVP (1.1) and (1.2) has exactly one solution. The proof is finished.

\section{Example}

Let us consider the following coupled system of fractional differential equations at resonance:

$$
\begin{cases}D_{0^{+}}^{3.5} u(t)=f\left(t, v, v^{\prime}, v^{\prime \prime}, v^{\prime \prime \prime}\right), & 0<t<1, \\ D_{0^{+}}^{3.6} v(t)=g\left(t, u, u^{\prime}, u^{\prime \prime}, u^{\prime \prime \prime}\right), & 0<t<1, \\ u(0)=u^{\prime}(0)=u^{\prime \prime}(0)=0, & u^{\prime \prime \prime}(0)=u^{\prime \prime \prime}(1) \\ v(0)=v^{\prime}(0)=v^{\prime \prime}(0)=0, & v^{\prime \prime \prime}(0)=v^{\prime \prime \prime}(1)\end{cases}
$$

where

$$
\begin{aligned}
& f\left(t, x_{1}, x_{2}, x_{3}, x_{4}\right)=\frac{t}{5}+\arctan x_{1}+\frac{1}{9} e^{-\left|x_{2}\right|}+\cos x_{3}+\frac{1}{8} x_{4}, \\
& g\left(t, y_{1}, y_{2}, y_{3}, y_{4}\right)=t^{2}+\frac{1}{4} \sin y_{1}+\sin ^{2}\left(y_{2} y_{3}\right)+\frac{1}{2}\left(y_{4}\right)^{\frac{1}{3}} .
\end{aligned}
$$


Corresponding to BVP (1.1) and (1.2), we have $\alpha=3.5$ and $\beta=3.6$. Take $a_{1}=\frac{59}{45}+\frac{\pi}{2}$, $a_{2}=\frac{9}{4}, b_{i}=c_{i}=0, i=1,2,3, b_{4}=\frac{1}{8}, c_{4}=\frac{1}{2}, \theta_{i}=\lambda_{i}=1, i=1,2,3, \theta_{4}=1, \lambda_{4}=\frac{1}{3}$, and $A=16$. Then we can calculate that $\left(\mathrm{H}_{1}\right)-\left(\mathrm{H}_{3}\right)$ hold.

\section{By Theorem 3.1, we see that BVP (4.1) has at least one solution.}

\section{Competing interests}

The authors declare that they have no competing interests.

\section{Authors' contributions}

All authors contributed equally to the writing of this paper. All authors read and approved the final manuscript.

\section{Author details}

${ }^{1}$ School of Science, Shandong Jiaotong University, Jinan, 250357, China. ${ }^{2}$ School of Science, China University of Mining and Technology, Beijing, 100083, China.

\section{Acknowledgements}

Research was supported by the Science Foundation of Shandong Jiaotong University (Z201429) and supported by the National Natural Science Foundation of China (11371364).

\section{Received: 20 January 2015 Accepted: 17 June 2015 Published online: 04 July 2015}

\section{References}

1. Kilbas, AA, Srivastava, HH, Trujillo, JJ: Theory and Applications of Fractional Differential Equations. Elsevier, Amsterdam (2006)

2. Oldham, KB, Spanier, J: The Fractional Calculus. Academic Press, New York (1974)

3. Podlubny, I: Fractional Differential Equations. Academic Press, New York (1999)

4. Weitzner, H, Zaslavsky, GM: Some applications of fractional equations. Commun. Nonlinear Sci. Numer. Simul. 8(3), 273-281 (2003)

5. Rui, W: Existence of solutions of nonlinear fractional differential equations at resonance. Electron. J. Qual. Theory Differ. Equ. 2011, 66 (2011)

6. Su, X: Boundary value problem for a coupled system of nonlinear fractional differential equations. Appl. Math. Lett. 22(1), 64-69 (2009)

7. Wang, G, Ahmad, B, Zhang, L: Impulsive anti-periodic boundary value problem for nonlinear differential equations of fractional order. Nonlinear Anal. 74(3), 792-804 (2011)

8. Agarwal, RP, O'Regan, D, Stanek, S: Positive solutions for Dirichlet problems of singular nonlinear fractional differential equations. J. Math. Anal. Appl. 371(1), 57-68 (2010)

9. Ahmad, B, Alsaedi, A: Existence and uniqueness of solutions for coupled systems of higher-order nonlinear fractional differential equations. Fixed Point Theory Appl. 2010, 364560 (2010)

10. Bai, Z: Solvability for a class of fractional m-point boundary value problem at resonance. Comput. Math. Appl. 62(3), 1292-1302 (2011)

11. Bai, Z, Zhang, Y: Solvability of fractional three-point boundary value problems with nonlinear growth. Appl. Math Comput. 218(5), 1719-1725 (2011)

12. Hu, Z, Liu, W, Chen, T: Existence of solutions for a coupled system of fractional differential equations at resonance. Bound. Value Probl. 2012, 98 (2012)

13. Hu, Z, Liu, W, Chen, T: Two-point boundary value problems for fractional differential equations at resonance. Bull. Malays. Math. Sci. Soc. (2) 36(3), 747-755 (2013)

14. Jafari, $\mathrm{H}, \mathrm{Gejii,}$ VD: Positive solutions of nonlinear fractional boundary value problems using Adomian decomposition method. Appl. Math. Comput. 180(2), 700-706 (2006)

15. Jiang, W: The existence of solutions to boundary value problems of fractional differential equations at resonance. Nonlinear Anal. 74(5), 1987-1994 (2011)

16. Xu, N, Liu, W, Xiao, L: The existence of solutions for nonlinear fractional multipoint boundary value problems at resonance. Bound. Value Probl. 2012, 65 (2012)

17. Zhang, Y, Bai, Z, Feng, T: Existence results for a coupled system of nonlinear fractional three-point boundary value problems at resonance. Comput. Math. Appl. 61(4), 1032-1047 (2011)

18. Zhang, Y, Bai, Z: Existence of solutions for nonlinear fractional three-point boundary value problems at resonance. J. Appl. Math. Comput. 36(1), 417-440 (2011)

19. Agarwal, RP, Benchohra, M, Hamani, S: Boundary value problems for fractional differential equations. Georgian Math J. 16(3), 401-411 (2009)

20. Kosmatov, N: Multi-point boundary value problems on an unbounded domain at resonance. Nonlinear Anal. 68(8), 2158-2171 (2008)

21. Kosmatov, N: A boundary value problem of fractional order at resonance. Electron. J. Differ. Equ. 2010, 135 (2010)

22. Mawhin, J: Topological degree and boundary value problems for nonlinear differential equations in topological methods for ordinary differential equations. In: Topological Degree and Boundary Value Problems for Nonlinear Differential Equations. Lect. Notes Math., vol. 1537, pp. 74-142. Springer, Berlin (1993) 